\title{
THE HOMOLOGY AND COHOMOLOGY GROUPS OF $\mathrm{H}_{3}$
}

\author{
Subrata Majumdar ${ }^{1}$ and Quazi Selina Sultana ${ }^{2}$ \\ ${ }^{12}$ Department of Mathematics, Rajshahi University, Rajshahi \\ E-mail:-majumdar_subrata@hotmail.com
}

Received 07.06.08

Accepted 27.06.09

\begin{abstract}
A free resolution of $\mathbf{Z}$ for the integral group ring of the three-dimensional Heisenberg group $H_{3}$ has been constructed by extending Lyndon's partial resolution. The integral homology and cohomology have been calculated from there.
\end{abstract}

AMS Classification: 18G, 20J.

Key words: Fox derivatives, free resolution.

\section{Introduction}

Here we shall construct a full free resolution of $\mathbf{Z}$ for the integral group ring of the three dimensional Heisenberg group, using Majumdar-Akhter [10] technique of extending Lyndon's partial free resolution [8] to a full free resolution. We compute the integral homology and cohomology from the resolution obtained.

$H_{3}$, the three dimensional Heisenberg group has a presentation

$$
H_{3}=\langle x, y, z:[x, z]=[y, z]=1,[x, y]=z\rangle \text {, (Burillo[1], p.2). }
$$

It is a member of widely studied important class of Lie Groups called the Heisenberg groups. It is nilpotent of class 2. Huebschmann [6] used his sophisticated perturbation theory technique to determine the cohomology of the generalized Heisenberg group given by

$$
\begin{aligned}
& G=\left\langle x, y, z:[x, z]=[y, z]=1,[x, y]=z^{k}\right\rangle . \\
& G=H_{3}, \text { if } \mathrm{k}=1 .
\end{aligned}
$$

The $(2 n+1)$ Heisenberg group $H_{2 n+1}$ is the group of upper triangular $(n+2) \times(n+2)$ matrices of the form:

$$
\left(\begin{array}{ccc}
1 & x & z \\
0 & I & y^{T} \\
0 & 0 & 1
\end{array}\right),
$$

where $x=\left(x_{1}, x_{2} \cdots, x_{n}\right), \mathrm{y}=\left(y_{1}, y_{2} \cdots, y_{n}\right), I$ is the $\mathrm{n} \times \mathrm{n}$ unite matrix. Thus $H_{3}$ is the group of all upper triangular matrices: 


$$
\left(\begin{array}{lll}
1 & x & z \\
0 & I & y \\
0 & 0 & 1
\end{array}\right),(x, y, z \in R)
$$

The Heisenberg group $H_{3}$ has a cubic Dehn function ([1], p.1) the latter being a best possible choice for isoperimetric function. Isoperimetric inequalities have been used fruitfully in the study of hyperbolic groups and automatic groups (Gromov [4], Epstien [2]). The cubic nature of Dehn function for $H_{3}$ shows that it is neither hyperbolic nor automatic, since these have respectively a linear Dehn function and a quadratic Dehn function. Thurston proved this fact by combinatorial methods. He also shows that $H_{3}$ is not combable

2. Before constructing our free resolution for $\mathrm{H}_{3}$, we give a few definitions, state a few known results and prove a number of results that will be needed for construction and its proof.

\section{Lemma 2.1}

$$
\mathrm{H}_{3} \text { is torsion free. }
$$

\section{Proof}

Now

$$
H_{3}=\left\{\left(\begin{array}{lll}
1 & x & z \\
0 & I & y \\
0 & 0 & 1
\end{array}\right):(x, y, z \in R)\right\} .
$$$$
\left(\begin{array}{ccc}
1 & x & z \\
0 & I & y \\
0 & 0 & 1
\end{array}\right)^{n}=\left(\begin{array}{ccc}
1 & n x & n z+n x y \\
0 & I & n y \\
0 & 0 & 1
\end{array}\right) \text {. }
$$

So

$$
\left(\begin{array}{lll}
1 & x & z \\
0 & I & y \\
0 & 0 & 1
\end{array}\right)^{n}=\left(\begin{array}{lll}
1 & 0 & 0 \\
0 & 1 & 0 \\
0 & 0 & 1
\end{array}\right),
$$

if and only if $n=0$.

Hence $\mathrm{H}_{3}$ is torsion free.

The following definitions are due to Higman [5].

\section{Definition 2.2}

A group $G$ is said to be indexed if it can be mapped homomorphically onto a non-zero subgroup of $\mathbf{Z}$.

\section{Definition 2.3}

A group $G$ is said to be indicable throughout if every subgroup $H(\neq 1)$ of $G$ can be indexed. 
We state two theorems due to Higmann:

Theorem 2.4 ([5], p.242)

If $G$ is indicable throughout and $R$ is a ring with, and has no zero divisors, then $R G$ has no zero divisors.

Theorem 2.5 ([5], p. 243)

If $G$ is indicable throughout and $R$ is a ring with $I$ and has no zero divisors, then the units of $R G$ are trivial.

We shall use these results to prove:

\section{Theorem 2.6}

$\mathrm{H}_{3}$ is indicable throughout.

\section{Proof}

We write $G$ for $H_{3}$ and let $H(\neq 1)$ be a subgroup of $G$.

\section{Case I}

First suppose that $H \subseteq G^{\prime}$. By the definition of $G, G^{\prime \prime} \subseteq Z(G)$. This implies that $G^{\prime}$ is abelian. $G^{\prime}$ is the normal subgroup generated by the commutator $\left[h_{1}, h_{2}\right], h_{1}, h_{2}$ are the images of $x_{1}, x_{2}$ in $G$. So a typical element $g^{\prime}$ of $G^{\prime}$ is $\Pi\left(g_{i}^{-1}\left(\left[h_{1}, h_{2}\right]^{e_{i}}\right)\right), e_{i}=1$.

$\therefore g^{\prime}=\prod_{i=1}^{n}\left[h_{1}, h_{2}\right]^{e_{i}}$, since $\left[h_{1}, h_{2}\right]$ is a commutator of $h_{1}$ and $h_{2}$.

Since $G^{\prime}$ is infinite cyclic, and since $H \neq 1, H$ too infinite cyclic. So $H$ can be indexed.

\section{Case II}

Suppose $H \subseteq G^{\prime}$. Then $H G^{\prime} \neq G^{\prime}$, and so, $\frac{H G^{\prime}}{G^{\prime}}$ is a subgroup of $\frac{G^{\prime}}{G}$, and is not the identity subgroup. Hence $\frac{H G^{\prime}}{G^{\prime}}$ is free abelian. Then there is a homomorphism $f: \frac{H G^{\prime}}{G^{\prime}} \rightarrow Z$ such that $\operatorname{Imf} \neq\{0\}$. If $\varphi$ is the canonical homomorphism $\varphi: H \rightarrow \frac{H G^{\prime}}{G^{\prime}}$, then $\varphi$ is onto and $\operatorname{Im} \bar{f} \neq\{0\}$, where $\bar{f}: H \rightarrow Z$ is the composite $\bar{f}=f \varphi$. Thus $H$ can be indexed.

Hence $G$ is indicable throughout.

As a consequence of Theorem 2.4, Theorem 2.5 and Theorem 2.6 implies the following:

\section{Corollary 2.7}

ZG has no zero divisors. 


\section{Corollary 2.8}

The units of $\mathbf{Z G}$ are trivial.

\section{Free resolution of $\mathbf{Z}$}

Let $G=H_{3}=\frac{F}{R}$, where $F$ is the free group generated by $x_{1}, x_{2}$ and $R$ is the normal closure of $r_{1}, r_{2}$, where $r_{1}=\left[x_{1},\left[x_{1}, x_{2}\right]\right]$ and $r_{2}=\left[x_{2},\left[x_{1}, x_{2}\right]\right]$.

i.e.,

$$
\begin{aligned}
& r_{1}=x_{1}^{-1} x_{2}^{-1} x_{1}^{-1} x_{2} x_{1} x_{1} x_{1}^{-1} x_{2}^{-1} x_{1} x_{2} \\
& r_{2}=x_{2}^{-1} x_{2}^{-1} x_{1}^{-1} x_{2} x_{1} x_{2} x_{1}^{-1} x_{2}^{-1} x_{1} x_{2} .
\end{aligned}
$$

Then the Fox derivatives of $r_{1}, r_{2}$ are:

$$
\begin{aligned}
& \frac{\partial r_{1}}{\partial x_{1}}=-r_{1}-x_{2} x_{1} r_{1}+x_{2}^{-1} x_{1} x_{2}+x_{2} \\
& \frac{\partial r_{1}}{\partial x_{2}}=-x_{1} r_{1}+x_{1} x_{2}^{-1} x_{1} x_{2}-x_{2}^{-1} x_{1} x_{2}+1 \\
& \frac{\partial r_{2}}{\partial x_{1}}=-x_{2}^{2} r_{2}+x_{2} x_{1}^{-1} x_{2}^{-1} x_{1} x_{2}-x_{1}^{-1} x_{2}^{-1} x_{1} x_{2}+x_{2} \\
& \frac{\partial r_{2}}{\partial x_{2}}=-r_{2}-x_{2} r_{2}+x_{2}^{-1} x_{1} x_{2}^{2} r_{2}+x_{1}^{-1} x_{2}^{-1} x_{1} x_{2}-x_{2}^{-1} x_{1} x_{2}+1
\end{aligned}
$$

Writing $\pi\left(x_{i}\right)=h_{i}, i=1,2$, we have

$$
\begin{aligned}
& \frac{\partial r_{1}}{\partial x_{1}}=-1-h_{2} h_{1}+h_{2}^{-1} h_{1} h_{2}+h_{2} ; \\
& \frac{\partial r_{1}}{\partial x_{2}}=-h_{1}+h_{1} h_{2}^{-1} h_{1} h_{2}-h_{2}^{-1} h_{1} h_{2}+1 ; \\
& \frac{\partial r_{2}}{\partial x_{1}}=-h_{2}^{2}+h_{2} h_{1}^{-1} h_{2}^{-1} h_{1} h_{2}-h_{1}^{-1} h_{2}^{-1} h_{1} h_{2}+h_{2} ; \\
& \frac{\partial r_{2}}{\partial x_{2}}=-1-h_{2}+h_{2}^{-1} h_{1} h_{2}^{2}+h_{1}^{-1} h_{2}^{-1} h_{1} h_{2}-h_{2}^{-1} h_{1} h_{2}+1 .
\end{aligned}
$$

To construct the free resolution of $\mathbf{Z}$ we proceed as follows. In Lyndon's partial free resolution of $\mathbf{Z}$, let $\beta_{1} \gamma_{1}+\beta_{2} \gamma_{2} \in \operatorname{Kerd}_{1}$, where $\gamma_{1}, \gamma_{2} \in Z G$.

Then $\quad d_{1}\left(\beta_{1} \gamma_{1}+\beta_{2} \gamma_{2}\right)=0$.

$\therefore\left[\alpha_{1}\left(h_{2}-1-h_{2} h_{1}-h_{2}^{-1} h_{1} h_{2}\right)+\alpha_{2}\left(1-h_{1}-h_{2}^{-1} h_{1} h_{2}+h_{1} h_{2}^{-1} h_{1} h_{2}\right)\right] \gamma_{1}$

$+\left[\alpha_{1}\left(-h_{2}^{2}-h_{2} h_{1}^{-1} h_{2}^{-1} h_{1} h_{2}-h_{1}^{-1} h_{2}^{-1} h_{1} h_{2}+h_{2}\right)+\alpha_{2}\left(h_{2}^{-1} h_{1} h_{2}^{2}+h_{1}^{-1} h_{2}^{-1} h_{1} h_{2}-h_{2}^{-1} h_{1} h_{2}-h_{2}\right)\right] \gamma_{2}$

$=0$ 
or,

$$
\begin{aligned}
& \left.\alpha_{1} \mid\left(h_{2}-1-h_{1} h_{2}-h_{2}^{-1} h_{1} h_{2}\right) \gamma_{1}+\left(-h_{2}^{2}-h_{2} h_{1}^{-1} h_{2}^{-1} h_{1} h_{2}-h_{1}^{-1} h_{2}^{-1} h_{1} h_{2}+h_{2}\right) \gamma_{2}\right\rfloor \\
& +\alpha_{2}\left[\left(1-h_{1}-h_{2}^{-1} h_{1} h_{2}+h_{1} h_{2}^{-1} h_{1} h_{2}\right) \gamma_{1}+\left(h_{2}^{-1} h_{1} h_{2}^{2}+h_{1}^{-1} h_{2}^{-1} h_{1} h_{2}-h_{2}^{-1} h_{1} h_{2}-h_{2}\right) \gamma_{2}\right]=0
\end{aligned}
$$

Since $Y_{0}$ is free on $\alpha_{1}, \alpha_{2}$, we have

$$
\begin{aligned}
& \left(h_{2}-1-h_{1} h_{2}-h_{2}^{-1} h_{1} h_{2}\right) \gamma_{1} \\
& +\left(-h_{2}^{2}-h_{2} h_{1}^{-1} h_{2}^{-1} h_{1} h_{2}-h_{1}^{-1} h_{2}^{-1} h_{1} h_{2}+h_{2}\right) \gamma_{2}=0 \\
& \left(1-h_{1}-h_{2}^{-1} h_{1} h_{2}+h_{1} h_{2}^{-1} h_{1} h_{2}\right) \gamma_{1} \\
& +\left(h_{2}^{-1} h_{1} h_{2}^{2}+h_{1}^{-1} h_{2}^{-1} h_{1} h_{2}-h_{2}^{-1} h_{1} h_{2}-h_{2}\right) \gamma_{2}=0
\end{aligned}
$$

We write Equation (3.1) as

$$
\left.\begin{array}{ll}
a \gamma_{1}+b \gamma_{2}=0 & (i) \\
c \gamma_{1}+d \gamma_{2}=0 & (i i)
\end{array}\right\} .
$$

Solving the equations (I) in $\mathbf{Q}$, we have $\gamma_{1}=\gamma_{1}^{\prime}$, where $\gamma_{1}^{\prime}$ is an arbitrary element of $\mathbf{Z G}$, and $\gamma_{2}=-b^{-1} a \gamma_{1}^{\prime}=-d^{-1} c \gamma_{1}^{\prime}$, so that $-b^{-1} a_{1}=-d^{-1} c . \gamma_{1}^{\prime}$ is an arbitrary element of ZG, $-b^{-1} a_{1}=-d^{-1} c \in Z G$. Since $b^{-1} a$ has a inverse $a^{-1} b, \quad b^{-1} a=g_{0}$, for some $g_{0} \in G$, by Corollary 2.8.

Define $Y_{2}$ as the right $\mathbf{Z G}$-module freely generated by $\delta$ and define

$$
\begin{gathered}
d_{2}: Y_{2} \rightarrow Y_{1} \text { by } \\
d_{2}(\delta)=\beta_{1}-\beta_{2} g_{0} .
\end{gathered}
$$

Then

$$
d_{2}\left(\delta \gamma_{1}\right)=\left(\beta_{1}-\beta_{2} g_{0}\right) \gamma_{1}=\beta_{1} \gamma_{1}-\beta_{2} g_{0} \gamma_{1}=\beta_{1} \gamma_{1}-\beta_{2} \gamma_{2} .
$$

Hence

$$
\beta_{1} \gamma_{1}+\beta_{2} \gamma_{2} \in \operatorname{Kerd}_{2} \text {. }
$$

$$
\left(d_{1} d_{2}\right)(\delta)=d_{1}\left(\beta_{1}-\beta_{2} g_{0}\right)
$$

Also

$$
\begin{aligned}
& =\left(\alpha_{1} a \gamma_{1}+\alpha_{2} a \gamma_{2}\right)-\left(\alpha_{1} b g_{0} \gamma_{1}+\alpha_{2} d g_{0} \gamma_{2}\right) \\
& =\alpha_{1}(a-a) \gamma_{1}+\alpha_{2}(c-c) \gamma_{2} \\
& =0
\end{aligned}
$$

$$
\therefore \operatorname{Kerd}_{2} \supseteq \operatorname{Im} d_{1} \text {. }
$$

Now let $\delta \in \operatorname{Kerd}_{2}$, then

$$
\begin{aligned}
& \alpha_{1} \gamma-\alpha_{2} g_{0} \gamma=0 \\
& \Rightarrow \gamma=0 .
\end{aligned}
$$

Thus we have 


\section{Theorem 3.1}

The following is a free $\mathbf{Z G}$-resolution of $\mathbf{Z}$ :

$$
0 \rightarrow Y_{2} \stackrel{d_{2}}{\longrightarrow} Y_{1} \stackrel{d_{1}}{\longrightarrow} Y_{0} \stackrel{d_{0}}{\longrightarrow} Z G \stackrel{\varepsilon}{\longrightarrow} Z \rightarrow 0,
$$

where $Y_{0}, Y_{1}, Y_{2}$ are right $\mathbf{Z G}$-modules freely generated by $\left\{\alpha_{1}, \alpha_{2}\right\},\left\{\beta_{1}, \beta_{2}\right\}, \delta$ and $\varepsilon, d_{0}, d_{1}, d_{2}$ are defined by

$$
\begin{aligned}
\varepsilon(g)=1, \forall g \in G ; & \\
d_{0}\left(\alpha_{1}\right)= & h_{1}-1 ; \\
d_{0}\left(\alpha_{2}\right)= & h_{2}-1 ; \\
d_{1}\left(\beta_{1}\right)= & \alpha_{1}\left(h_{2}-1-h_{2} h_{1}-h_{2}^{-1} h_{1} h_{2}\right)+\alpha_{2}\left(1-h_{1}-h_{2}^{-1} h_{1} h_{2}+h_{1} h_{2}^{-1} h_{1} h_{2}\right) \\
& d_{1}\left(\beta_{2}\right)=\alpha_{1}\left(-h_{2}^{2}-h_{2} h_{1}^{-1} h_{2}^{-1} h_{1} h_{2}-h_{1}^{-1} h_{2}^{-1} h_{1} h_{2}+h_{2}\right) \\
& +\alpha_{2}\left(h_{2}^{-1} h_{1} h_{2}^{2}+h_{1}^{-1} h_{2}^{-1} h_{1} h_{2}-h_{2}^{-1} h_{1} h_{2}-h_{2}\right) . \\
d_{2}(\delta)= & \beta_{1}-\beta_{2} g_{0} .
\end{aligned}
$$

\section{Homology}

For a left ZG-module $A$, the homology groups $H_{n}(G, A)$ are given by the homology of the complex of abelian groups

$$
0 \rightarrow A \stackrel{\overline{d_{2}}}{\longrightarrow} A^{2} \stackrel{\overline{d_{1}}}{\longrightarrow} A^{2} \stackrel{\overline{d_{0}}}{\longrightarrow} A \rightarrow 0,
$$

where $\overline{d_{0}}, \overline{d_{1}}, \overline{d_{2}}$ are given by

$$
\begin{aligned}
& \overline{d_{0}}\left(a_{2}, a_{2}\right)=\left(h_{1}-1\right) a_{1}+\left(h_{2}-1\right) a_{2} ; \\
& \overline{d_{1}}\left(a_{1}, a_{2}\right)=\left(\left[h_{2}-1-h_{2} h_{1}-h_{2}^{-1} h_{1} h_{2}\right] a_{1},\right. \\
& \left.\left[-h_{2}^{2}-h_{2} h_{1}^{-1} h_{2}^{-1} h_{1} h_{2}-h_{1}^{-1} h_{2}^{-1} h_{1} h_{2}+h_{2}\right] a_{2}\right) \\
& \overline{d_{2}}(a)=\left(a, g_{0} a\right) .
\end{aligned}
$$

If $A$ is trivial, then

$$
\begin{aligned}
& \overline{d_{0}}\left(a_{2}, a_{2}\right)=0 ; \\
& \overline{d_{1}}\left(a_{1}, a_{2}\right)=(0,0) ; \\
& \overline{d_{2}}(a)=(a, a) .
\end{aligned}
$$

If $A=Z$, then 


$$
\begin{aligned}
& H_{0}(G, Z) \cong Z, \\
& H_{1}(G, Z) \cong Z \oplus Z, \\
& H_{2}(G, Z) \cong Z, \\
& H_{3}(G, Z) \cong 0 .
\end{aligned}
$$

\section{Cohomology}

For a right ZG-module $A$, the cohomology groups $H^{n}(G, A)$ are given by the homology of the complex

$$
0 \leftarrow A \stackrel{d_{2}^{*}}{\longleftarrow} A^{2} \stackrel{d_{1}^{*}}{\longleftarrow} A^{2} \stackrel{d_{0}^{*}}{\longleftarrow} A \leftarrow 0,
$$

where $d_{0}^{*}, d_{1}^{*}, d_{2}^{*}$ are given by

$$
\begin{aligned}
& d_{0}^{*}(a)=\left(a\left(h_{1}-1\right), a\left(h_{2}-1\right)\right) ; \\
& d_{1}^{*}\left(a_{1}, a_{2}\right)=\left(a_{1}\left[h_{2}-1-h_{2} h_{1}-h_{2}^{-1} h_{1} h_{2}\right], a_{2}\left[-h_{2}^{2}-h_{2} h_{1}^{-1} h_{2}^{-1} h_{1} h_{2}-h_{1}^{-1} h_{2}^{-1} h_{1} h_{2}+h_{2}\right]\right), \\
& d_{2}^{*}(a)=a_{1}-g_{0} a_{1} .
\end{aligned}
$$

If $A$ is trivial, then

$$
\begin{aligned}
& d_{0}^{*}(a)=(0,0) ; \\
& d_{1}^{*}\left(a_{1}, a_{2}\right)=(0,0) \\
& d_{2}^{*}(a)=0 .
\end{aligned}
$$

If $A=Z$, then

$$
\begin{aligned}
& H^{0}(G, Z) \cong Z, \\
& H^{1}(G, Z) \cong Z \oplus Z, \\
& H^{2}(G, Z) \cong Z \oplus Z, \\
& H^{3}(G, Z) \cong Z .
\end{aligned}
$$

\section{REFERENCES}

1. Burillo J., Lower bound isoperimetric function for nilpotent groups, Dimacs series in discrete Mathematics and theoretical computer science 25, 1-8, (1996).

2. Epstien A. B. D., J. W. Cannon, D. F. Holt, S. V. F. Levy, M. S. Peterson, W. P. Thurston, Word processing in groups, Jones and Bartlett, Boston -London. (1992).

3. Fox R. H., Free Differential Calculus I, Ann. Math. 57, 3,547-560, (1953).

4. Groves J. R. J., Rewriting systems and Homology of groups, Proceedings of the third International conference on the theory of Groups, Canberra 1456 LNM, Springer-Verlag, (1989). 
5. Higman G., The zero divisors and units in a group ring, Proc. Lond. Math. Soc. 46(2), 231-248, (1940).

6. Huebschmann J., Perturbation theory and free resolutions for nilpotent groups of class 2, J. Algebra, 126 2, 348-399, (1984).

7. Lyndon R. C. and P. Schupp, Combinatorial group theory, Springer-Verlag, Berlin, (1997).

8. Lyndon R. C., Cohomology theory of groups with a single defining relation, Ann. Math. 52, 3, 656-665, (1953).

9. Majumdar S. and N. Akhter, On a simple method of determining the homology and the cohomology for finitely presented group. Bull. Cal. Math. Soc. 100, (2008).

1. Majumdar S., A free resolution for a class of groups, J. Lond.Math. Soc. 2, 615-619, (1970). 